\title{
First PAC experiments in MAX-phases
}

\author{
D. Jürgens • M. Uhrmacher • H. Hofsäss • J. Röder • \\ P. Wodniecki · A. Kulinska • M. Barsoum
}

Published online: 6 August 2008

(C) The Author(s) 2008

\begin{abstract}
MAX-phases are hexagonal ternary carbides and nitrides with the general formula: $M_{n+1} A X_{n}$ and $n=1$ to $3 .{ }^{111}$ In was implanted into the two MAX compounds $\mathrm{Ti}_{2} \mathrm{InC}$ and $\mathrm{Zr}_{2} \mathrm{InC}$. Based on the general knowledge of previous ${ }^{111}$ In implantations one expects to find the probes on the indium lattice-site in these compounds. First experiments on the annealing behaviour and the thermal stability of the indiumcontaining MAX-phases are reported. The observed EFGs are interpreted and first PAC-measurements under compressive stress are shown.
\end{abstract}

Keywords Perturbed angular correlation (PAC) $\cdot \mathrm{MAX}$-phase $\cdot \mathrm{Ti}_{2} \mathrm{InC} \cdot \mathrm{Zr}_{2} \mathrm{InC} \cdot$ Static pressure

\section{Introduction}

MAX-phases are layered, hexagonal ternary carbides and nitrides (general formula: $M_{n+1} A X_{n}$ where $n$ varies from 1 to 3 ). $M$ stands for an early transition metal, A for an A-group (mostly IIIA and IVA) element and $X$ represents either $\mathrm{C}$ and/or $\mathrm{N}$. They belong to space group $\mathrm{D}_{6 \mathrm{~h}}^{4}, \mathrm{P}_{3} / \mathrm{mmc}$, with two formula units per unit cell (see Fig. 1). $X$-ions sit in the centre of an $M$-octahedron. The 211 MAX-phases

D. Jürgens · M. Uhrmacher $(\bowtie) \cdot$ H. Hofsäss

II. Physikalisches Institut, Universität Göttingen,

Friedrich-Hund-Platz 1, 37077 Göttingen, Germany

e-mail: muhrmac@gwdg.de

J. Röder

Institut für Physikalische Chemie, TU Braunschweig, Braunschweig, Germany

P. Wodniecki · A. Kulinska

IFJPAN, 31-342 Krakow, Poland

M. Barsoum

Dep. Mat. Sci. +Eng., Drexel University, Philadelphia, PA 19104, USA 
Fig. 1 Structure of $\mathrm{M}_{2} \mathrm{AX}$ (=211): $M$ gray, $A$ white, $X$ black

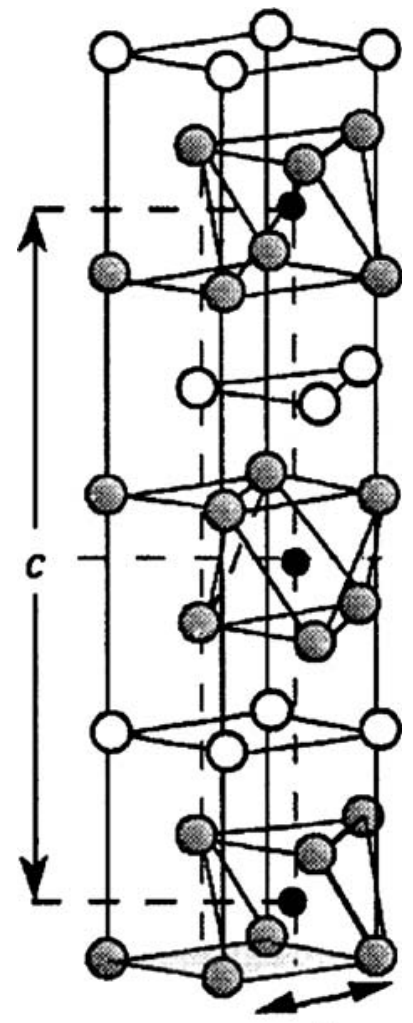

a

$(n=1)$ show in the direction of the $c$-axis the layer-sequence $A M X M A \ldots$, the 312 phases $(n=2)$ show $A M X M X M A \ldots$ and the 413 phases $(n=3)$ come with AMXMXMXMA....

These compounds combine some of the best properties of metals and ceramics. Like metals, they are electrically and thermally conductive, most readily machinable, not susceptible to thermal shock, plastic at high temperatures, and exceptionally damage tolerant. Like ceramics, they are elastically rigid, lightweight, and maintain their strengths to high temperatures. The ternaries $\mathrm{Ti}_{3} \mathrm{SiC}_{2}$ and $\mathrm{Ti}_{2} \mathrm{AlC}$ are creep, fatigue and oxidation resistant [1]. At present the explanation of this extraordinary behavior is assumed to be in the microstructure of the layered material: kinking bands and delamination seem to play a central role [2].

Using perturbed $\gamma \gamma$-angular correlations (PAC) with ${ }^{111}$ In probe nuclei, changes of MAX-phase properties during elastic deformations can be observed on an atomic scale. PAC experiments in complex compounds with different crystallographic sites often suffer from the problem to find the position of the probes. In the two MAX compounds $\mathrm{Ti}_{2} \mathrm{InC}$ and $\mathrm{Zr}_{2} \mathrm{InC}$ the probe ${ }^{111} \mathrm{In}$ is a constituting element of the compounds. Therefore, one expects to find the probes on the indium lattice-site, which should establish a PAC fingerprint, typically for the In-site in MAX phases, or more general for the $A$-site in the MAX compounds. In complete analogy, the $M$-site fingerprint can be discovered using the ${ }^{181} \mathrm{Hf}$ probe. Such studies will provide 
Fig. 2 Pressed $\mathrm{Ti}_{2} \mathrm{InC}-$ seen with an electron raster microscope. The typical nanolaminate structure of a MAX-phase clearly shows up

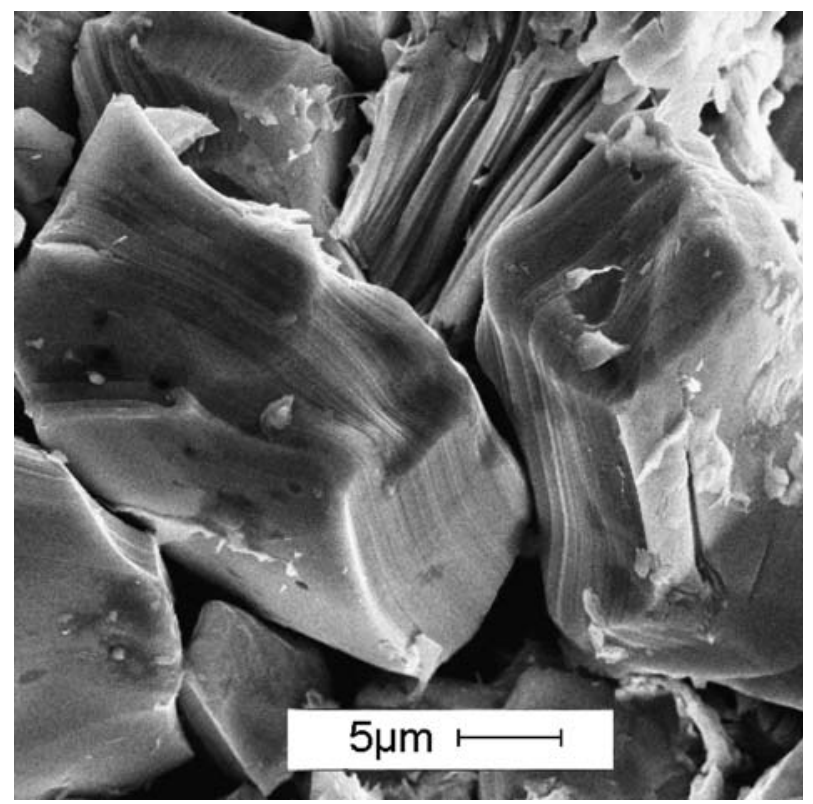

the key information to investigate by PAC the microstructure of the full class of MAX phases (about 50 compounds) which do not necessarily contain In-ions on the $A$-site or Hf-ions on the $M$-site of the structure. Knowing the "fingerprints" the technical important MAX-phases can be investigated.

\section{Experimental details}

The $\mathrm{Ti}_{2} \mathrm{InC}$ and $\mathrm{Zr}_{2} \mathrm{InC}$ samples have been provided by $\mathrm{M}$. Barsoum. The fabrication is described in Ref. [3]: Elemental powders of $\mathrm{Zr}$, Ti, In and graphite have been mixed in the proper stoichiometric ratios and cold pressed at $630 \mathrm{MPa}$. The resulting cylindrical pellets were sealed in borosilicate glass tubes under vacuum, placed in a hot isostatic press (HIP), heated at $20^{\circ} \mathrm{C} / \mathrm{min}$ to $973 \mathrm{~K}$ and held at that temperature for $30 \mathrm{~min}$, before Ar was introduced to the chamber. The HIP was then heated at the same rate to $1573 \mathrm{~K}$, where it was held for $7 \mathrm{~h}$ before cooling. The pressure at $1573 \mathrm{~K}$ was $\sim 50 \mathrm{MPa}$. Finally the glass was removed mechanically. Predominantly single phase, fully dense samples were obtained. They were cut by a diamond saw into $20 \mathrm{~mm}^{2}$ large slices of about $1 \mathrm{~mm}$ thickness.

About $10^{12}$ of ${ }^{111} \mathrm{In}^{+}$ions were implanted at $400 \mathrm{keV}$ into such samples, using the Göttingen implanter IONAS [4]. The radiation damage after the implantation was annealed out by heating the samples above $T_{\mathrm{a}}=700 \mathrm{~K}$ in vacuum, which caused some problems (see Section 3.1). PAC-spectra were taken at different measuring temperatures $T_{\mathrm{m}}$, with the help of a standard setup of four NaI-detectors in $90^{\circ}$ geometry. Details on the data analysis can be found in [5]. The static pressure was applied by placing a sample between two DURAL-pistons, which were screwed against each other (Fig. 2). A pressure of about $1 \mathrm{GPa}$ could be estimated from the "engrammes" made by the sample in the Al. 


\section{Results}

\subsection{Indium loss}

In first studies it was reported, that $\mathrm{Ti}_{2} \mathrm{InC}$ evaporates indium, when heated in vacuum at $1,173 \mathrm{~K}$ for $\sim 2 \mathrm{~h}$. XRD showed the emergence of peaks corresponding to $\mathrm{TiC}_{x}$. Also a weight loss was found. It was assumed that $\mathrm{Ti}_{2} \mathrm{InC}$ dissociates peritectically into the A-group element and the $\mathrm{MX}_{2}$ phase [3]. Some light on the final dissociation product came from FPLAPW-calculations of ordered titanium carbide $\left(\mathrm{Ti}_{2} \mathrm{C}\right)$-phases. The trigonal phase was found to be more stable than the cubic one, but all calculated $\mathrm{Ti}_{2} \mathrm{C}$ phases were found to be stable against segregation into $\mathrm{TiC}$ and metallic $\mathrm{Ti}$ [6]. The observation of In-whisker formation on $\mathrm{Zr}_{2}$ InC samples can be seen in the same context: The samples contained some unreacted indium in the grain boundaries. The In-content, determined from differential scanning calorimetric analysis, was 4 vol. \%. The majority of the grains ranged in the size between 3-5 $\mu \mathrm{m}$ [7].

Heating $\mathrm{Ti}_{2} \mathrm{InC}$ for 1 day at $473 \mathrm{~K}$ and for 1 day at $573 \mathrm{~K}$ in vacuum (PACtempering sequence) caused a loss of about of $30 \%$ of the implanted ${ }^{111} \mathrm{In}$ probes. Less pronounced was this loss for $\mathrm{Zr}_{2} \mathrm{InC}$ under similar conditions, and for $\mathrm{Ti}_{2} \mathrm{InC}$ it could be lowered by a vacuum annealing for only $10 \mathrm{~min}$ at $873 \mathrm{~K}$.

\subsection{The MAX-phase $\mathrm{Ti}_{2} \mathrm{InC}$}

PAC spectra after the implantation (Fig. 3, top) show the need for an annealing step, although some ${ }^{111}$ In-loss can be expected (Section 3.1). Afterwards a fraction $f_{\text {In }} \sim 50 \%$ of the probes were found at the substitutional site in In-metal precipitates, identified by the well known frequency of $17 \mathrm{MHz}$ with $\eta=0$ [8]. When heating the sample above the melting point of indium $(430 \mathrm{~K})$, the precipitates transform into liquids, which have no EFG. Consequently, a PAC measurement at $T_{\mathrm{m}}=436 \mathrm{~K}$ shows now the fraction $f_{\text {In }} \sim 50 \%$ with $v_{\mathrm{Q}}=0 \mathrm{MHz}$. This process is reversible and proves the existence of In-precipitates, un-reacted indium or decomposed MAXphase. The rest of the probes has a well defined EFG, fitted with the parameters $v_{\mathrm{Q}}=290 \mathrm{MHz}$ and $\eta=0$. Spectra are given in Fig. 3, the temperature and annealing conditions are given in the same figure. XRD analyses before and after the PAC cycle showed the compound still intact and gave no hints of disintegration phases. Therefore, this high frequency is attributed to probes at the In-lattice site (A-site) of $\mathrm{Ti}_{2} \mathrm{InC}$.

\subsection{The MAX-phase $\mathrm{Zr}_{2} \mathrm{InC}$}

The necessary vacuum annealing step after the ${ }^{111}$ In-implantation caused-as expected-also in this MAX-compound a loss of indium. The contaminated container/envelope was removed and also this compound showed $65 \%$ of the probes with the typical parameters of the substitutional site in In-metal $\left(v_{\mathrm{Q}}=17 \mathrm{MHz}\right.$, $\eta=0$ ). Above the indium melting point we found $f_{\mathrm{In}}=65 \%$ with $v_{\mathrm{Q}}=0 \mathrm{MHz}$, a clear proof, that the ${ }^{111} \mathrm{In}$ is located in metallic indium. The rest of the probes showed two 

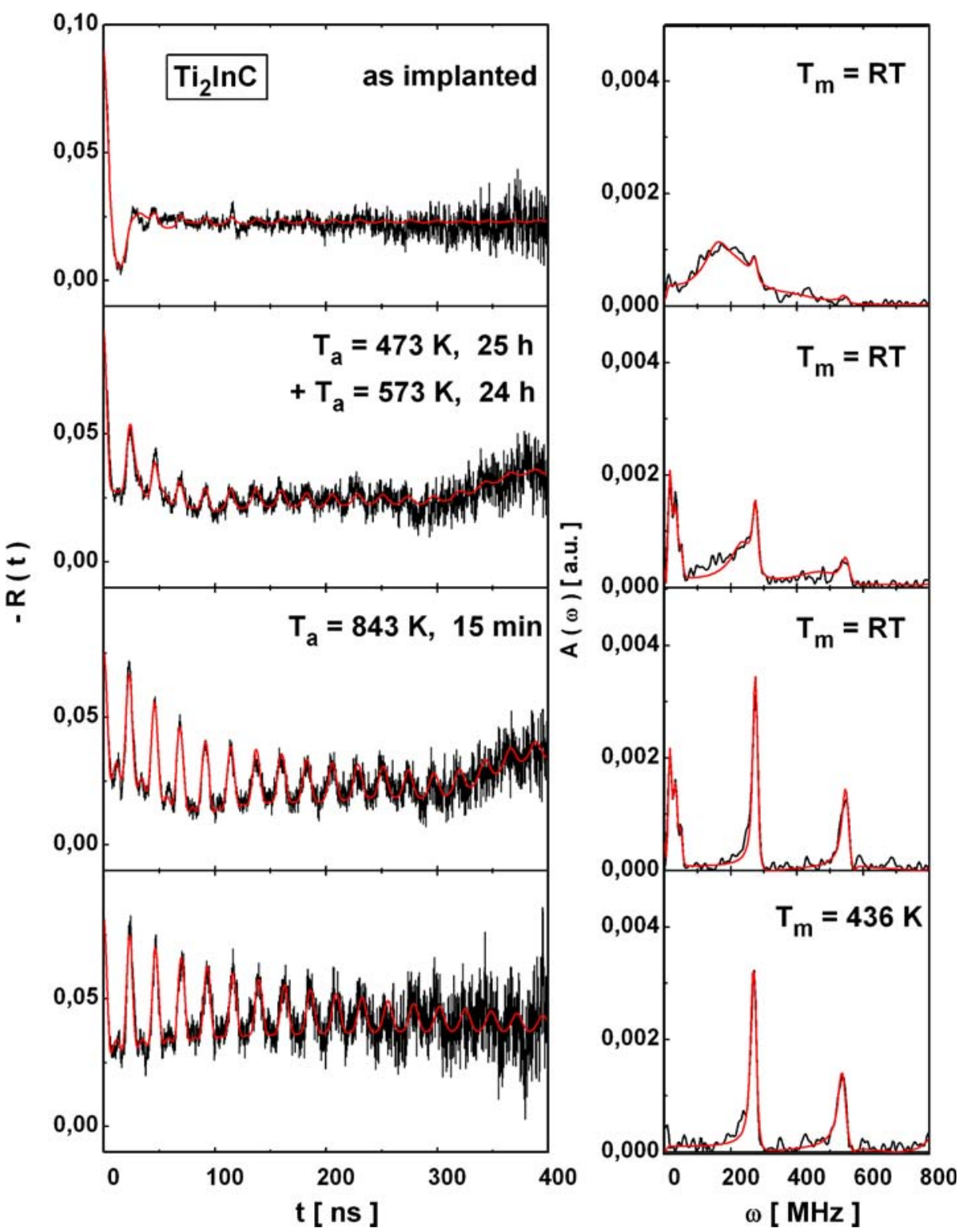

Fig. 3 PAC-spectra and their Fourier transforms for $\mathrm{Ti}_{2} \mathrm{InC}$

well defined EFGs of similar magnitude, fitted with the parameters $v_{\mathrm{Q} 1}=348 \mathrm{MHz}$, $v_{\mathrm{Q} 2}=328 \mathrm{MHz}$ and $\eta_{1}=\eta_{2}=0$ (see Table 1). The PAC-spectra are given in Fig. 4. The temperature and annealing conditions are included in the same figure. Similarly to the case of $\mathrm{Ti}_{2} \mathrm{InC}$ we attribute this high frequency to probes on the In-lattice site of $\mathrm{Zr}_{2} \operatorname{InC}$. 
Table 1 PAC parameters of the A-site EFG (measured at RT) in different MAX-phases: $v_{\mathrm{Q}}$ and $\eta$ describe the strength and symmetry of the EFG, $\delta$ is the distribution-width around $\nu_{\mathrm{Q}}$ and the fraction gives the percentage of probes on this A-site

\begin{tabular}{lllll}
\hline Compound & $v_{\mathrm{Q}}[\mathrm{MHz}]$ & $\eta$ & $\delta[\mathrm{MHz}]$ & Fraction [\%] \\
\hline $\mathrm{Ti}_{2} \mathrm{InC}$ & $290(3)$ & 0 & $6(1)$ & $43-65$ \\
$\mathrm{Zr}_{2} \mathrm{InC}$ & $348(3)$ & 0 & $2(0.3)$ & 25 \\
& $328(9)$ & 0 & $5(1)$ & 12.5 \\
$\mathrm{Ti}_{3} \mathrm{SiC}_{2}$ & $348(3)$ & 0 & $2(0.3)$ & 68 \\
\hline
\end{tabular}
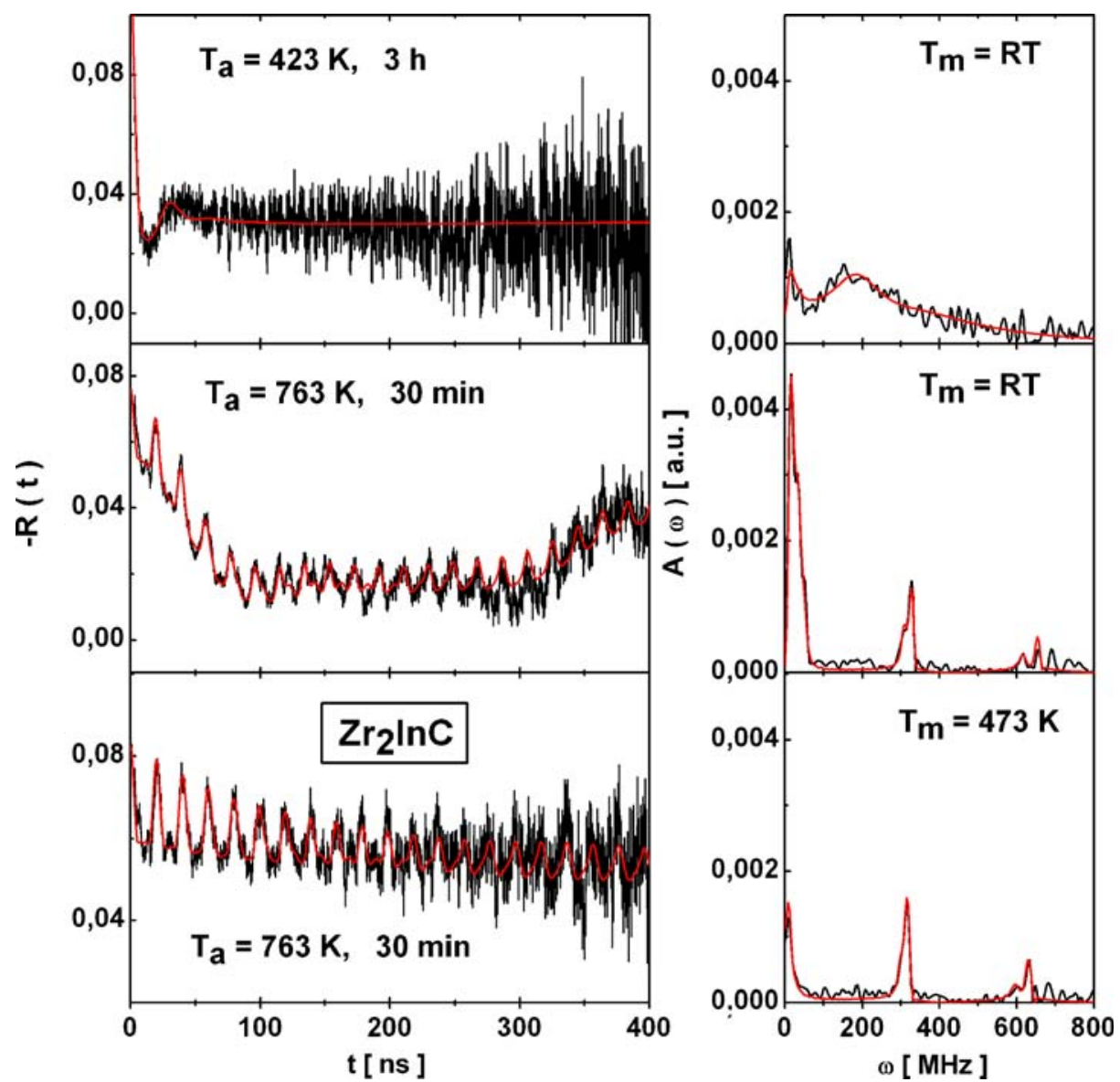

Fig. 4 PAC-Spectra and their Fourier transforms for $\mathrm{Zr}_{2} \mathrm{InC}$

\subsection{Compressive stress on $\mathrm{Ti}_{2} \mathrm{InC}$}

After all annealing steps, which prepared a maximum of the A-site EFG (Fig. 5, upper part), the $\mathrm{Ti}_{2} \mathrm{InC}$ sample of Fig. 3 was placed between two Al-pistons. The sample's position was flat in the detector plane. Under compressive stress, the PAC 

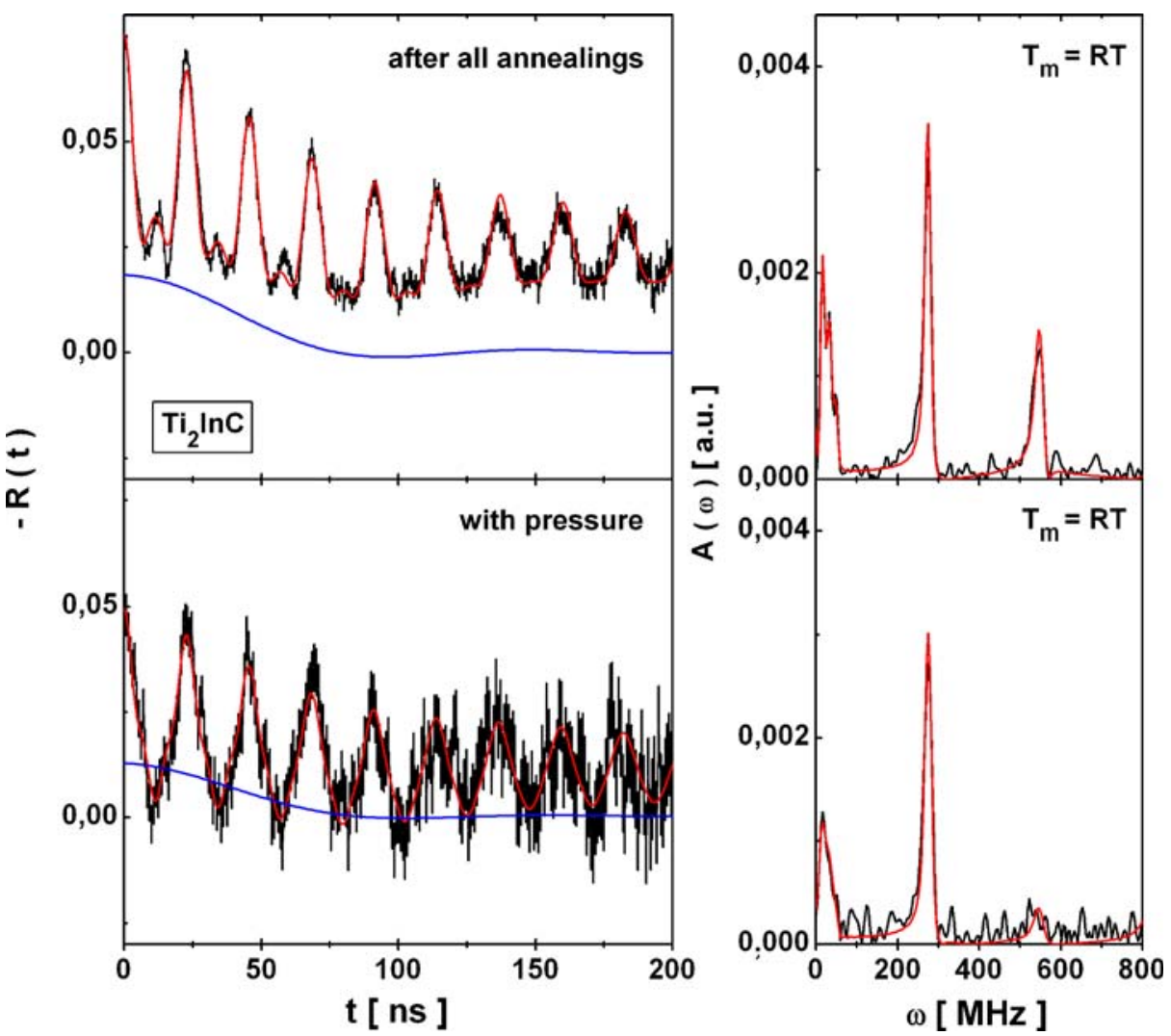

Fig. $5 \mathrm{Ti}_{2}$ InC well annealed (upper panel), under pressure (lower panel). The line shows the part of the $R(t)$-function, which is caused by probes in metallic indium

spectrum (Fig. 5, lower part) showed a clear texture with the EFG pointing into the detector plane, i.e. vertically to the direction of the pressure.

\section{Discussion}

The idea to find a "fingerprint EFG" of the A-site in MAX phases seems to work. In Table 1 the observed high frequencies are collected, including a first result on $\mathrm{Ti}_{3} \mathrm{SiC}_{2}$, probably the technologically most important MAX-phase, which is stable to high temperatures and contains no indium in the structure. In all three of them we observed high EFGs in the range between 290 and $350 \mathrm{MHz}$. As demonstrated in the oxides [9] different compounds of the same lattice structures (as an example bixbyite class: $\mathrm{In}_{2} \mathrm{O}_{3}, \mathrm{Y}_{2} \mathrm{O}_{3}, \ldots$ ) show similar EFGs for a probe on a specific site in that structure. Despite the problematic question of a disintegration of the In-containing MAX phases, we assume that these new high EFGs are typical for probes on the A-site in the MAX phases.

All three compounds show a different result, therefore carbon-precipitates can be excluded, as they should give the same frequency in all three cases. Nevertheless, 
the identical frequency $v_{\mathrm{Q}}=348 \mathrm{MHz}$ is observed in two different compounds. The strength of the observed EFGs is similar to impurity-In pairs in semiconductors [10]. We propose, that the ${ }^{111}$ In probe-sitting on the A-site-catches an interstitial carbon atom. The small distance within this pair causes the high EFG. A trapping in slightly different geometries might explain, why in $\mathrm{Zr}_{2} \mathrm{InC}$ two very similar high EFGs are observed. Unfortunately, this hypothesis can not yet be proven, but has to be solved in the future.

One of the extraordinary features of the MAX-phases is the elastic behavior of these ceramics. Our first simple compression experiment shows, that the PAC technique is sensitive to this question. Having the probe on a well known site, we will be able to learn more about the microscopic changes during application and release of pressure in these layered structures.

Acknowledgement A part of the study was supported by the BMBF under contract 05KK7MG1.

Open Access This article is distributed under the terms of the Creative Commons Attribution Noncommercial License which permits any noncommercial use, distribution, and reproduction in any medium, provided the original author(s) and source are credited.

\section{References}

1. Barsoum, M.W., Zhen, T., Kalidindi, S., Radovic, M., Murugaiah, A.: Nat. Mater. 2, 107-111, 291 (2003)

2. Barsoum, M.W., Farber, L., El-Raghy, T.: Met. Mater. Trans. 30A, 1727-1738 (1999)

3. Barsoum, M.W., Golczewski, J., Seifert, H.J., Aldinger, F.: J. Alloys Compd. 340, 173-179 (2002)

4. Uhrmacher, M., et al.: Nucl. Instrum. Methods B139, 306 (1998)

5. Aldon, L., Uhrmacher, M., Branci, C., Ziegeler, L., Roth, J., Schaaf, P., Metzner, H., OlivierFourcade, J., Jumas, J.C.: Phys. Rev., B 58, 11303-11312 (1998)

6. Eibler, R.: J. Phys. Condens. Matter 14, 4425-4444 (2002)

7. Barsoum, M.W., Hoffmann, E.N., Doherty, R.D., Gupta, S., Zavaliangos, A.: Phys. Rev. Letts. 93, 206104-1 (2004)

8. Witthuhn, W., Engel, W.: In: Christiansen, J. (ed.) Hyperfine Interactions of Radioactive Nuclei, p. 272. Springer, Berlin (1983)

9. Uhrmacher, M.: Physica. B 389, 58-66 (2007)

10. Tessema, G., Vianden, R.: J. Phys. Condens. Matter 15, 5297-5306 (2003) 